\title{
Fault Diagnosis in Dynamic Systems Using Fuzzy Interacting Observers
}

\author{
N. V. Kolesov \\ Concern Central Scientific and Research Institute ELEKTROPRIBOR, JSC, State Research Center of the Russian Federation, \\ 30 Malaya Posadskaya Street, Saint Petersburg 197046, Russia
}

Correspondence should be addressed to N. V. Kolesov; kolesovnv@mail.ru

Received 12 September 2012; Accepted 1 January 2013

Academic Editor: Sendren Sheng-Dong Xu

Copyright (c) 2013 N. V. Kolesov. This is an open access article distributed under the Creative Commons Attribution License, which permits unrestricted use, distribution, and reproduction in any medium, provided the original work is properly cited.

A method of fault diagnosis in dynamic systems based on a fuzzy approach is proposed. The new method possesses two basic specific features which distinguish it from the other known fuzzy methods based on the application of fuzzy logic and a bank of state observers. First, this method uses a bank of interacting observers instead of traditional independent observers. The second specific feature of the proposed method is the assumption that there is no strict boundary between the serviceable and disabled technical states of the system, which makes it possible to specify a decision making rule for fault diagnosis.

\section{Introduction}

A dynamic system model is widely used to describe technical systems in solving various problems of analysis and synthesis, including diagnosis, as applied to these systems. Although the literature on problems of diagnosis is abundant, the interest to them still persists and the investigations are continuing. The problems of how to increase accuracy, or diagnostic depth, and how to take into account different uncertainties that are inherent in the solution of diagnosis problems are conventionally central to the studies of fault diagnosis. It is to these problems that this paper is devoted, wherein the technical states of the system are assumed uncertain.

In the literature, the diagnosis problem is considered in different formulations, depending primarily on the models used to describe a system: deterministic [1-8], stochastic $[9,10]$, fuzzy [11-13], and so forth. The choice of a formulation is determined, as a rule, by the application and the problem to be solved by the dynamic system, as well as a priori information on the properties of the system and its possible faults available to developers of diagnostic tools. Thus, if a developer possesses statistical information on the system behavior and faults, it makes sense to use the stochastic approach. If such information is unavailable, the deterministic approach is preferential because in this case, information on uncertainties is minimal. The fuzzy approach complements the tools of the deterministic approach with the rules of analysis and fault decision making based on fuzzy logic. These rules formalize the empirical knowledge of the developer about the nominal and anomalous (in the case of faults) behavior of a system and uncertainties inherent in this problem by introducing fuzzy sets. Of course, there is a certain analogy between the methods that belong to different approaches. It is a consequence of mutual penetration and enrichment of the existing approaches. In this sense, the material presented later is not an exception.

Each of the afore mentioned approaches fits different lines of investigations. Among the most efficient of them is the one that relies on the models of the diagnosed system for synthesis of diagnostic tools. This line is developed in the framework of all approaches and is realized by application of either single state observers [3-5] (stochastic approachKalman and Wiener filters $[9,10]$ ) or output observers $[2,3]$, or their sets (banks) [7-13] as parts of diagnostic tools. If we use banks, each of the observers $O_{i}$ (Figure 1) is adjusted to one of the technical states of the system (serviceable $S_{0}-O_{0}$, disabled $S_{1}$ with the first fault $O_{1}$, disabled $S_{2}$ with the second fault $\mathrm{O}_{2}$, etc.). In the general case, vector residuals (difference signals) $v_{0}, v_{1}, \ldots, v_{N}$ between the real output of the dynamic system and the output of each of the observers are formed, 


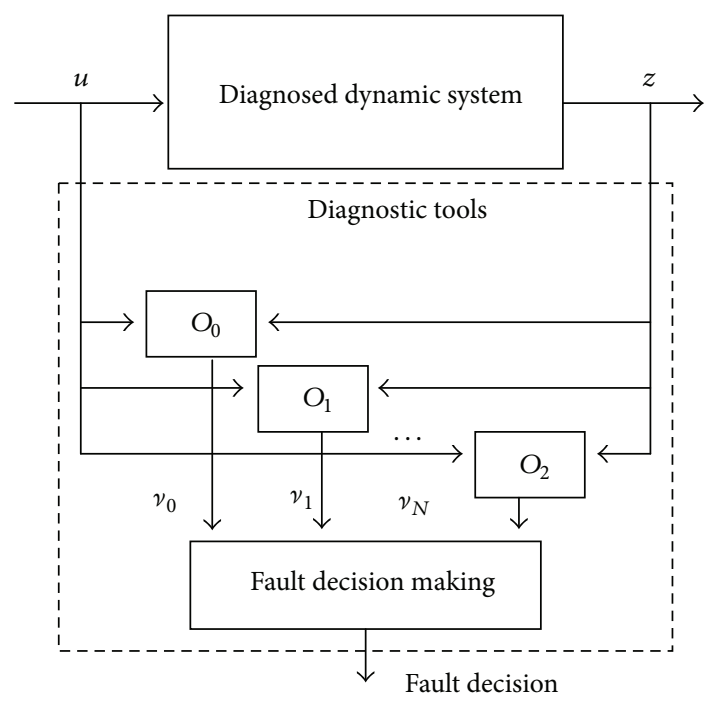

Figure 1: Structure of the diagnostic system.

which is followed by a decision making on the technical state of the diagnosed system.

In this study, we propose a method for diagnosing dynamic systems in the framework of a fuzzy approach. The new method possesses two basic specific features which distinguish it from the other known fuzzy methods based on the application of fuzzy logic and a bank of state observers. First, unlike the approaches in which observers are independent of each other, in this method, we consider a bank of interacting observers. The second specific feature of the proposed method is an assumption that there is no strict boundary between the serviceable and disabled technical states of the system and its elements, which makes it possible to specify a decision making rule for fault diagnosis.

\section{Preliminary Definitions and Remarks}

First of all, let us discuss the notion of a "fuzzy" technical state and, as a consequence, "fuzzy" fault, which is understood as a transition from the serviceable technical state to the disabled one. The notion of a fuzzy technical state used later seems to be quite adequate to describe the existing engineering approach. Indeed, judging from the value of the parameter which indicates the technical state of an object, an engineer can conclude that the object is either serviceable or disabled. Depending on a particular value of this parameter, the engineer can conclude that the object is serviceable or, correspondingly, disabled to a certain extent.

We define a fuzzy technical state of an object with respect to parameter $\Theta$ as a linguistic variable characterized, for example, by two terms (fuzzy sets) - serviceable and disabled technical states described by the corresponding membership functions $\mu_{i}^{0}$ and $\mu_{i}^{1}$.

Figure 2 illustrates the notions of "crisp" (a) and "fuzzy" (b) technical states of an object. In the first case, the domains of values of the key parameter $\Theta$ corresponding to the serviceable and disabled technical states of an object (denoted by rectangles of different colors) are separated by a strict boundary. In the second case, these domains intersect (shaded area), and they are determined by the corresponding membership functions with parameters $a$ and $b$. As a result, for any value of $\Theta=\Theta^{\prime}$, the technical state of the object can be related both with a fuzzy set of serviceable $\left(\mu_{i}^{0}=0.8\right)$ and a fuzzy set of disabled $\left(\mu_{i}^{1}=0.3\right)$ states. Note that in this paper, we only consider trapezoidal membership functions:

$$
\begin{aligned}
& \mu_{i}^{0}= \begin{cases}1, & 0 \leq \Theta_{i} \leq a, \\
\frac{b-\Theta_{i}}{b-a}, & a \leq \Theta_{i} \leq b, \\
0, & \text { otherwise, }\end{cases} \\
& \mu_{i}^{1}= \begin{cases}0, & 0 \leq \Theta_{i} \leq a, \\
\frac{\Theta_{i}-a}{b-a}, & a \leq \Theta_{i} \leq b, \\
1, & \text { otherwise. }\end{cases}
\end{aligned}
$$

We should also make two more remarks concerning the problems touched upon in this paper. First, for simplicity, we assume that there are no perturbations in the considered model of the system. Second, we do not discuss the already known procedures of synthesis of stable observers [14].

\section{Decision Making Rule on Fault Occurrence}

The rule is based on the notion of the confidence coefficient $K_{i}$ of the $i$ th technical state introduced later. It requires that the confidence coefficient $K_{i}$ should reach a specified level Afor a technical state with the dominant value of this coefficient; that is, the following condition should be satisfied:

$$
K^{*}=\max _{i}\left\{K_{i}\right\} \geq A .
$$

Let us clarify the procedure of calculation of confidence coefficient. It is based on two groups of parameters that define the technical state of a dynamic system: the residuals $v_{i}, i=$ $\overline{0, N}$ obtained as a result of comparison of the system outputs with the outputs of the observers and the estimates $\widehat{\delta}_{i}, \widehat{\Delta}_{i}$, $i=\overline{0, N}$ of the levels of the faults in the diagnosis in the signal space and parameter space, respectively. It is assumed that the residual $v_{i}, i=\overline{0, N}$ formed as a result of comparison of the system outputs with the $i$ th observer can be represented by a linguistic variable, for example, with two terms, "small" and "large," for which the membership functions $\mu_{v_{i}}^{0}$ and $\mu_{v_{i}}^{1}$, $i=\overline{0, N}$ are defined. The term "small' corresponds to the situation in which the model used in the observer synthesis is adequate to the current technical state of the diagnosed system. The occurrence of a small, as it is, but nonzero value of this residual is due to the transient processes accompanying the estimation, the lack of complete adequacy of the model of the diagnosed system used for the observer synthesis, and neglected perturbations of its dynamics and output. The term "large" corresponds to the situation in which the model used in the observer synthesis is essentially inadequate to the current technical state of the diagnosed system. This is the case when, for example, a diagnosed system is in the $i$ th 


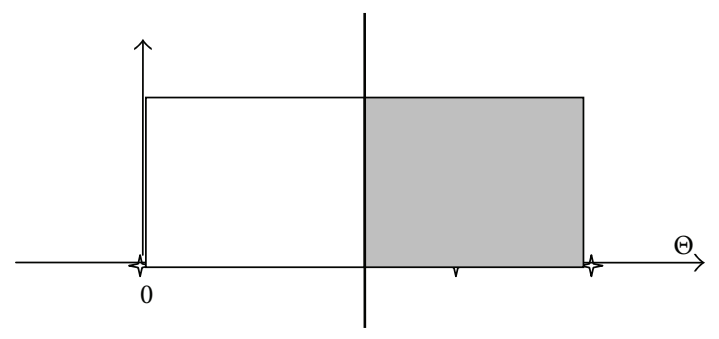

(a)

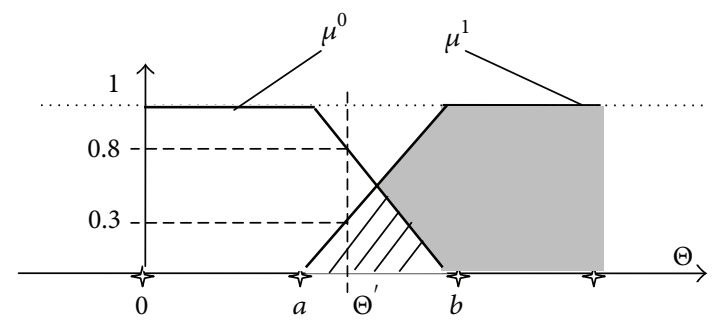

(b)

FIgURE 2: Illustration of the ideas of "crisp" and "fuzzy" technical states.

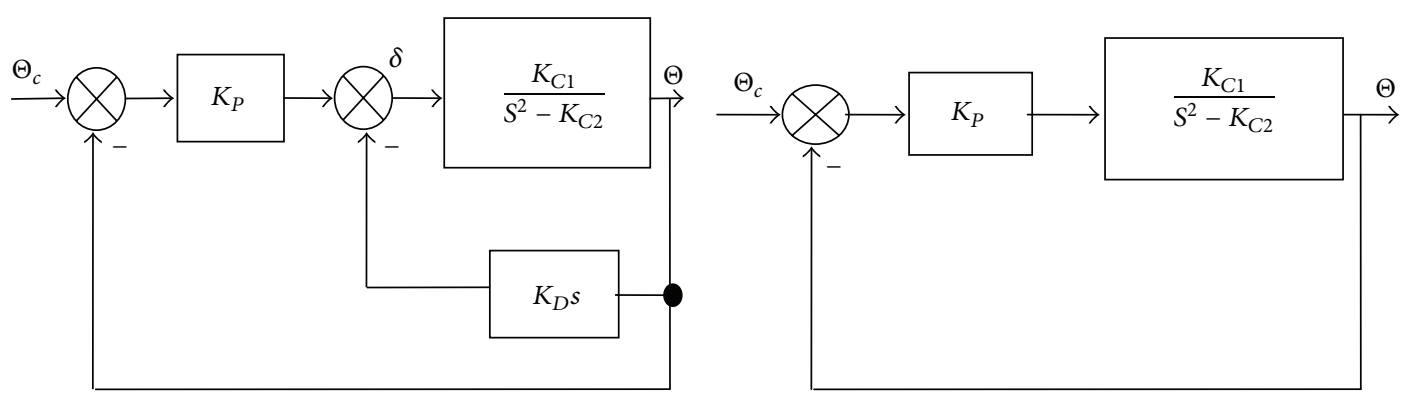

(a)

(b)

FIGURE 3: Structure of an attitude control system of a space launch vehicle (a) and an example of structural changes (b).

technical state, whereas the observer is adjusted for the $j$ th technical state. In this case, parameters $\left\{a_{i}, b_{i} \mid i=\overline{0, N}\right\}$ of the membership functions are determined by the equalities:

$$
a_{i}=\min _{i}\left\{v_{i} \mid S_{j}, j \neq i\right\}, \quad b_{i}=\max _{i}\left\{v_{i} \mid S_{j}, j=i\right\} .
$$

As for the variables $\widehat{\delta}_{i}, \widehat{\Delta}_{i}, i=\overline{0, N}$ simulating a fault, we also assume that they are described by linguistic variables with two terms, "serviceable" and "disabled," with the membership functions $\mu_{\hat{\delta}_{i}}^{0}$ and $\mu_{\widehat{\delta}_{i}}^{1}, i=\overline{1, N}$, or $\mu_{\hat{\Delta}_{i}}^{0}$ and $\mu_{\widehat{\Delta}_{i}}^{1}, i=\overline{1, N}$, respectively, specified for them.

In order to obtain the confidence coefficients $\left\{K_{i} \quad \mid\right.$ $i=\overline{0, N}\}$, first, we determine the characteristics called generalized membership degrees $\left\{\tilde{\mu}_{i} \mid i=\overline{0, N}\right\}$ of the technical state of the diagnosed system to each of the possible fuzzy technical states. These characteristics generalize the information on the technical state of the system with respect to all observers and are formed based on the set of values $\left\{v_{i} \mid i=\overline{0, N}\right\}$. The value of the generalized membership degree is formed in accordance with the following expression:

$$
\widetilde{\mu}_{i}=\mu_{v_{i}}^{0} \mu_{\overbrace{\widehat{\delta}_{i}}}^{1} \prod_{\substack{j=0 \\ j \neq i}}^{N} \mu_{v_{j}}^{1} .
$$

The explanation of this expression is obvious. Indeed, the observer adequate to the technical state of the system forms a small residual, whereas the others form large residuals. This being so, we may say that a fault does exist if $\delta_{i}$ is large. We do not consider the situations including equivalent or poorly distinguishable faults.

Then, the confidence coefficient $K_{i}$ for each technical state $S_{i}$ is calculated in accordance with the rule of "weighting coefficients" by determining the contribution of the generalized membership degree $\tilde{\mu}_{i}$ to the sum of these degrees for all states. Consider

$$
K_{i}=\frac{\widetilde{\mu}_{i}}{\sum_{j=0}^{N} \widetilde{\mu}_{j}} .
$$

\section{Diagnosis of Structural Changes}

In this paper, we consider successively three different models of fault: structural changes, faults in the signal space, and faults in the parameter space. In so doing, we study various structures of diagnostic tools different in the organization of the banks of the state observers and decision making rules. The proposed structures are compared with the known variant of diagnostic tools, which uses a bank of independent observers (Figure 1), and a decision is made as a result of fuzzy analysis of residuals. First, consider the diagnosis of structural changes. A structure of a system is very convenient to describe such faults. An example of a system structure is given in Figure 3(a). It is an attitude control system of a space launch vehicle (LV). The angular position $\Theta$ is adjusted by changing angle $\delta$, which characterizes the inclination of the engines relative to the LV's axis. An example of structural changes is a break of the velocity feedback (Figure 3(b)).

From here on, the diagnosed dynamical system is described in the time domain (by differential or difference 
equations); that is, for a linear system,

$$
\dot{x}(t)=F x(t)+G u(t), \quad y(t)=H x(t),
$$

while for a nonlinear system,

$$
\dot{x}(t)=\varphi(x(t), u(t), \Theta), \quad y(t)=H x(t),
$$

where $x$ is an $n$-dimensional state vector, $u$ is an $m$ dimensional input vector, $y$ is a $p$-dimensional output vector, $F$ is an $n \times n$ matrix of dynamic, $G$ is an input $n \times m$ matrix, $H$ is an output $p \times n$ matrix, $\varphi$ is a function of dynamic, and $\Theta$ is a parameter vector.

The problem of synthesis of tools for fault diagnosis involves two main questions: formation of a rule for decision making and synthesis of a bank of observers. Assuming that the answer to the first question is given in the previous section, we proceed to the discussion of the second question.

Let us next study some variants of fault diagnosis that employ the banks of both independent and interacting observers. By this we mean the method used to form the system state vector estimate in each of the observers. If it is formed independently, we have independent observers and treat the obtained estimates as conditional with respect to a certain technical state. If the formation of the estimate also takes into account the estimates obtained in other observers, we have interacting observers. This chapter is referring to the independent observers.

Let us consider briefly the question of synthesis of a single observer. Observers may be synthesized by different rules, resulting from differences in the formulation of the problem. The procedure for the synthesis of a state observer for the linear system is known; however, for the sake of completeness, we will recall its main issues. As previously stated, each $i$ th observer of the bank is adjusted to a technical state of the system, in which it is characterized by matrices $F_{i}, G_{i}, H_{i}$. The matrices $F_{i}^{*}, G_{i}^{*}, H_{i}^{*}$ of the corresponding observer will be the same. The observer equations for diagnostics of the linear system have the form [14]

$$
\begin{gathered}
\dot{x}_{i}^{*}(t)=F_{i}^{*} x_{i}^{*}(t)+G_{i}^{*} u(t)+L_{i}\left(y-y_{i}^{*}\right), \\
y_{i}^{*}(t)=H_{i}^{*} x_{i}^{*}(t) .
\end{gathered}
$$

As a result, an $i$ th fault that occurs in the diagnosed system during operation leads to formation of an estimate of the system state vector in the observer. In this case, the behavior of the estimate error $e_{i}(t)=x_{i}(t)-x_{i}^{*}(t)$ is determined by the equation $\dot{e}_{i}=\left(F_{i}^{*}-L_{i} H_{i}^{*}\right) e_{i}$. It will suffice to determine the feedback matrix $L_{i}$ to synthesize the observer. It is determined depending on the desired behavior of the estimation error. Undoubtedly, this error must tend to zero. Therefore, matrix $F_{i}^{*}-L_{i} H_{i}^{*}$ corresponding to each of the observers must be stable; that is, the real parts of its eigenvalues must take on negative values. For the case when the original system is linear, stationary, and observable, the selection algorithm of matrix $L_{i}$ is known, and it is rather simple [14]. It uses the description of the diagnosed dynamical system in identification canonical form such that if the diagnosed system has one output, it is a single chain of integrators with feedback from the last integrator. Such system representation allows to find matrix $L_{i}$ based on desired eigenvalues for the matrix $F_{i}^{*}-L_{i} H_{i}^{*}$. If the diagnosed system has many outputs, the procedure for determining the feedback matrix is complicated [14], although the sequence of steps is the same. In a similar way, first of all, we need to present the diagnosed system in the identification canonical form, which becomes more complicated. It contains not a single chain of integrators with feedback from the last integrator, but several-as many as there are outputs in the system.

To summarize this section, we formulate the algorithm for diagnosis of arbitrary faults in the formulation under consideration.

\section{Algorithm 1.}

(1) Formation of a list of faults.

(2) Synthesis of the independent observer for each of the faults.

(3) Assignment of membership functions for the considered fuzzy residuals based on the developer's empirical knowledge of the system operability.

(4) Decision making about faults by forming a confidence coefficient.

To illustrate the proposed algorithm, once again, we turn to the example in Figure 3.

Example 2. Let us synthesize the diagnosis tools for break of the velocity feedback. The nominal behavior of the system is described by the equation

$$
\ddot{\Theta}-K_{D} K_{C 1} \dot{\Theta}+\left(K_{P}+K_{C 1}-K_{C 2}\right) \Theta=K_{P} K_{C 1} \Theta_{c},
$$

but in the case of fault, it is described as follows:

$$
\ddot{\Theta}+\left(K_{P}+K_{C 1}-K_{C 2}\right) \Theta=K_{P} K_{C 1} \Theta_{c} .
$$

We analyzed an intermittent fault, when a device recovers after the failure and then fails again, in the Simulink environment using a sinusoidal input signal. In practice, faults of this type usually are a severe problem for diagnosis. Simulation results of a problem of diagnosis are shown in Figure 4, which presents time diagrams for the confidence coefficients formed with the use of a bank of two independent observers adjusted for nominal and faulty states, respectively. It is easy to see that the diagnosis tools show adequate results.

\section{Diagnosis in Signal Space}

In the case of diagnosis in the signal space, the fault is simulated as an additional term $\delta$ in the dynamics equation; that is, for an initial linear system, we have

$$
\dot{x}(t)=F x(t)+G u(t)+\delta, \quad y(t)=H x(t),
$$

and for a nonlinear system,

$$
\dot{x}(t)=\varphi(x(t), u(t), \Theta)+\delta, \quad y(t)=H x(t),
$$




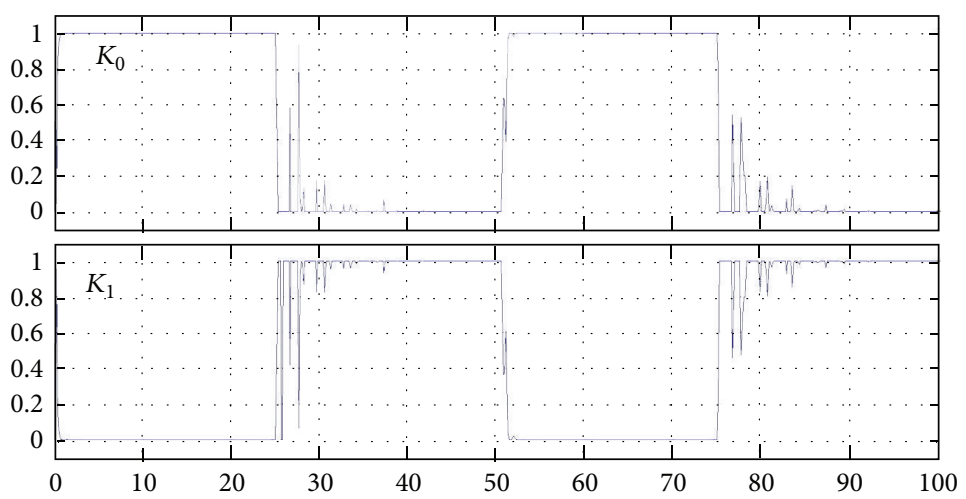

FIGURE 4: Time diagrams for confidence coefficients of technical states of an attitude control system of a space launch vehicle for independent observers.

where $x$ is the $n$-dimensional state vector, $u$ is the $m$ dimensional output vector, $y$ is the $p$-dimensional output vector, $F$ is the $n \times n$ dynamics matrix, $G$ is the input $n \times m$ matrix, $H$ is the output $p \times n$ matrix, $\varphi$ is the dynamics function, and $\Theta$ is the parameter vector. In this case, the number of types $N$ of single faults is equal to the dimension $n$ of the state vector of the diagnosed system. The first type is simulated by an additional term in the first dynamics equation for the first component of vector $x$ and the second type in the second equation for the corresponding component of vector $x$, and so forth. Faults within the same type are distinguished by the level of term $\delta$.

In this case of diagnosis in the signal space, the state vector $x_{i}^{*}$ of the observer $O_{i}(i=\overline{0, N})$ is formed by adding to the state vector $x$ of the diagnosed system of the variable $\delta_{i}$ simulating fault; that is, $x_{i}^{*^{T}}=\left[x^{T} \delta_{i}^{T}\right]$. Taking into account the assumption that variable $\delta_{i}$ is constant, the equation for this variable takes the form

$$
\dot{\delta}_{i}=0
$$

As a result, in the presence of the $i$ th fault, the matrices of the diagnosed system take the forms

$$
\begin{gathered}
F_{i}=\left[\begin{array}{cc} 
& 0 \\
F_{0} & \cdots \\
& 1 \\
\cdots \\
0 \cdots 0 & 0
\end{array}\right], \quad G_{i}=\left[\begin{array}{c}
G_{0} \\
0 \cdots 0
\end{array}\right], \\
H_{i}=\left[\begin{array}{cc} 
& 0 \\
H_{0} & \cdots \\
& 0
\end{array}\right],
\end{gathered}
$$

where in the dynamics matrix, the unity in the last column is in the $i$ th row. If the $i$ th fault occurs in the diagnosed system in the course of its operation, the estimate of this compound vector is formed in the observer, as well as the estimate $\widehat{\delta}_{i}$ of the value of variable $\delta_{i}$.
Let us discuss the proposed method for obtaining an estimate of the state vector of the system in each of the observers. Further, consider two diagnostic algorithms with the application of a bank of interacting observers. In so doing, we use the decision making rule on the fault occurrence described in the previous section. It will be shown later that in the general case, the efficiency of the considered algorithms is higher than that in the case of independent observers.

An important specific feature of the first algorithm is that on each successive step of the calculation, each of the observers is based on the estimate of state $\hat{x}(t)$ obtained as a result of averaging of partial estimates of all the observers determined at the previous step rather than the independently formed partial estimate $\widehat{x}_{i}(t)$ of the state vector. Here, the current values of the confidence coefficients serve as weighting coefficients, where

$$
\widehat{x}(t)=\sum_{i} K_{i} \widehat{x}_{i}(t)
$$

The result of it is a nonlinear state feedback. Indeed, the residual (estimation error) formed by an adequate observer tends to zero, and the corresponding confidence coefficient increases with the decrease of confidence coefficients for other technical states. Thus, in expression (15), the relative weight of the estimate formed in an adequate observer increases.

The second algorithm, though being similar to the previous one, differs from it, first of all, in the fact that the observers are matched not with technical states but with the transitions between them. In this case, it is assumed that the observer is matched with the transition $S_{i} \rightarrow S_{j}$ if it is based on the state estimate obtained under condition $S_{i}$ and was synthesized based on the model of the system in state $S_{j}$. In this case, transitions of $S_{i} \rightarrow S_{i}$ type, that is, the transitions that do not change the technical state, are also taken into account among the analyzed transitions. As a result, the confidence coefficients (denoted by $K_{i j}$ ) calculated in this algorithm according to the rule from the previous section correspond to 

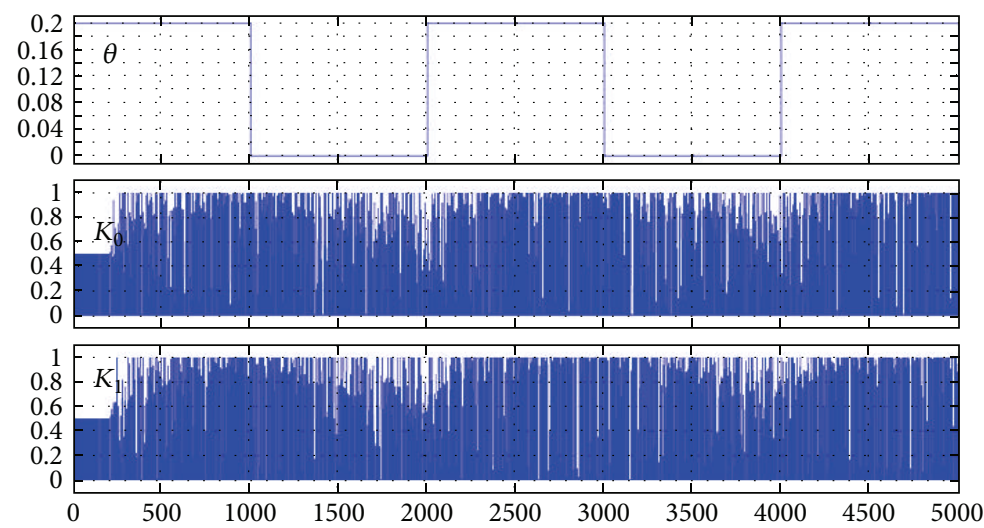

(a)
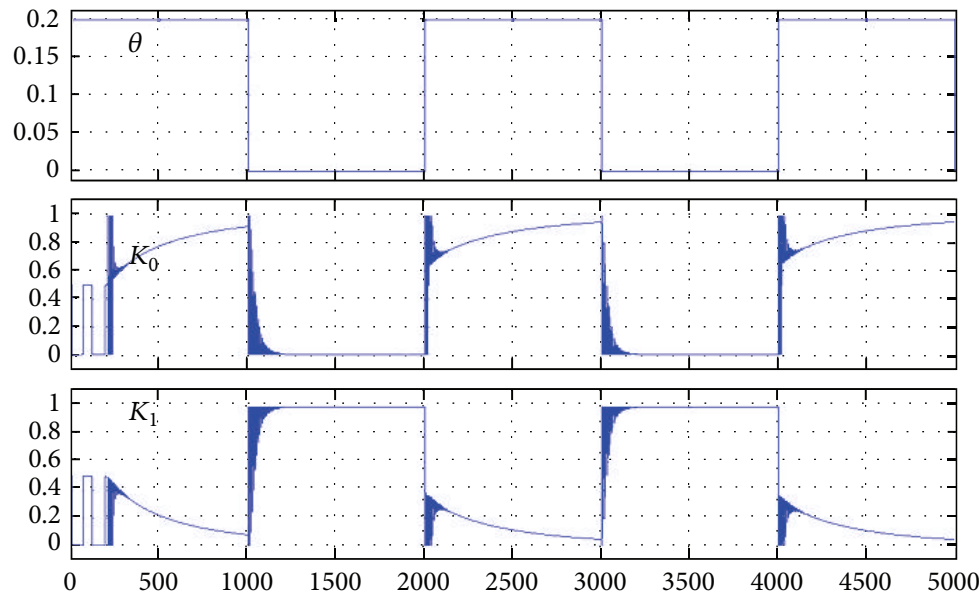

(b)

FIGURE 5: Time diagrams for alternating fault and confidence coefficients of technical states of the control system for (a) independent and (b) interacting observers.

the transitions $S_{i} \rightarrow S_{j}$ between technical states, rather than technical states themselves. This being so, at any step for each technical state $S_{j}$, we should form the conditional estimate $\widehat{x}_{j}(t)$ following the rule

$$
\widehat{x}_{j}(t)=\sum_{i} K_{i j} \hat{x}_{i j}(t) .
$$

In order to make a decision according to rule (2), we need to determine the confidence coefficients $K_{i},(i=\overline{0, N})$ for the technical

$$
K_{i}=\sum_{j=0}^{N} K_{i j}
$$

It is evident that the analysis of the behavior of the diagnosed system used in the second algorithm is more detailed, which is why we can expect this method to be more efficient. In the general case, this is proved by the simulation results given later.

Let us illustrate the described algorithms by a particular example.
Example 3. Let us consider the linear system characterized by the matrices

$$
\begin{aligned}
& F=\left[\begin{array}{ccccc}
-0,0061 & 0,5122 & -0,0579 & 0,029 & 0,0377 \\
-0,5122 & -0,1868 & 0,6803 & -0,1417 & -0,2028 \\
-0,0579 & -0,6803 & -0,7645 & 0,7531 & 0,8508 \\
-0,29 & -0,1417 & -0,7531 & -0,3258 & -0,5974 \\
-0,0377 & -0,2028 & -0,8508 & -0,5974 & -1,242
\end{array}\right], \\
& G=\left[\begin{array}{c}
0,0452 \\
0,2335 \\
0,2779 \\
0,09742 \\
0,1329
\end{array}\right] \\
& H=\left[\begin{array}{llllll}
0,0452 & -0,2334 & 0,2779 & -0,09743 & -0,1329
\end{array}\right] \text {. }
\end{aligned}
$$

This system is a reduced model of an aircraft control loop at an altitude obtained by linearization of the aircraft motion equations in the neighborhood of the nominal trajectory. This description covers the controlled object, the rudder control servo drive, the altitude sensor, and the controller. For this example, the problem of diagnosis in the signal space was 

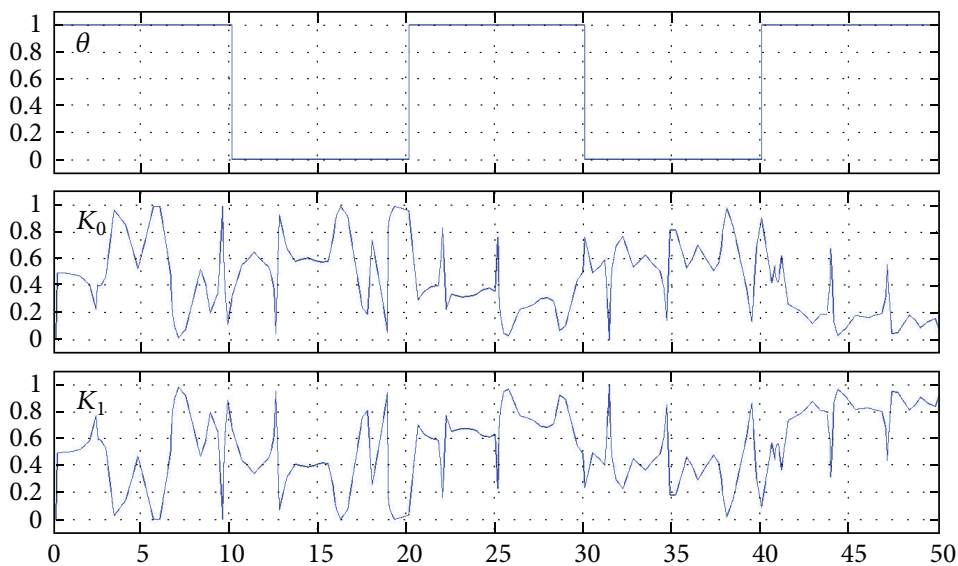

(a)
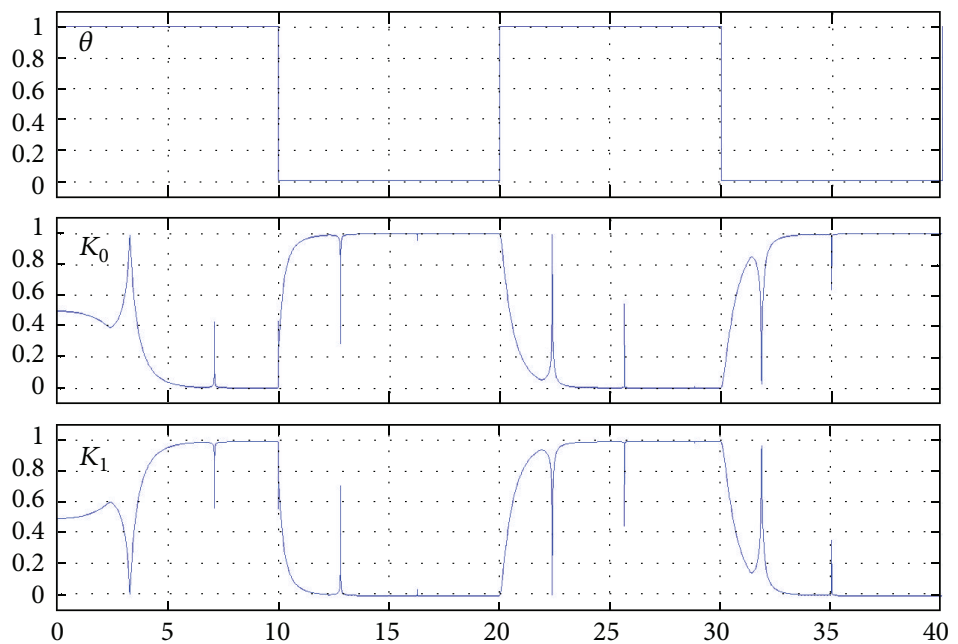

(b)

FIGURE 6: Time diagrams for alternating fault and confidence coefficients of technical states of a torpedo for (a) independent and (b) interacting observers.

simulated in Simulink. An intermittent fault $\theta$ in the form of a meander was simulated at the first integrator (first diagram in Figures 5(a) and 5(b)). A sinusoidal signal with amplitude of 0.5 was fed to the input of the system. Figure 5 shows the time diagrams of the obtained confidence coefficients for the cases of independent and interacting observers. It is clear that in the case of independent observers (Figure 5(a)), the diagnostic tools do not form the expected result, since before the fault occurs, coefficient $K_{0}$ should take a stable value close to unity, and coefficient $K_{1}$ a value close to zero. After the fault, the coefficient $K_{1}$ tends to the value close to unity, and $K_{0}$ to a value close to zero. When we use interacting observers (Figure 5(b)) matched with technical states, the time diagrams demonstrate an adequate operation of the diagnostic tools. Thus, on the time interval before a fault occurs, the confidence coefficient $K_{0}$ for a serviceable technical state after the transient process connected with the initial estimation takes a value equal to unity. After the fault, the corresponding confidence coefficient takes a stable value close to unity.

\section{Diagnosis in Parameter Space}

In the case of diagnosis in the parameter space, the fault is simulated as the deviation of the value of some system parameter from the nominal value. Thus, for example, for the linear system, it is simulated as the deviation of elements of the system matrices from the nominal, where

$$
\begin{gathered}
\dot{x}(t)=F(\Theta+\Delta \Theta) x(t)+G(\Theta+\Delta \Theta) u(t), \\
y(t)=H(\Theta+\Delta \Theta) x(t),
\end{gathered}
$$

and for the nonlinear system,

$$
\dot{x}(t)=\varphi(x(t), u(t), \Theta+\Delta \Theta), \quad y(t)=H x(t) .
$$

In this case, the number of types $N$ of single faults is equal to the number of system parameters.

Let us use the algorithms considered in the previous section in the diagnosis in the parameter space. Assume that the parameter $\Theta$ is a diagnosed parameter. Let us divide 
the interval of the parameter values into $l$ subintervals $\left\{\Theta_{i}+\right.$ $\left.\Theta_{i+1} \mid i=\overline{1, l}\right\}$. Let us match the observer based on the system model for $\left\{\Theta_{i} \mid i=\overline{1, l}\right\}$ with each of the subintervals. We take into account that in this case, observers do not form the estimate of the fault value directly; however, it can be obtained according to the expression $\widehat{\Delta}=\Theta_{i^{*}}-\Theta_{\text {nom }}$, where $i^{*}=\arg \min _{i} v_{i}, \Theta_{\text {nom }}$ is the nominal value of the parameter $\Theta$.

Let us analyze the efficiency of the diagnostic algorithms using the following example.

Example 4. Let us consider the model of a water torpedo described by the nonlinear equation

$$
J \ddot{\varphi}+C_{1} \dot{\varphi}=-C_{2} C_{3} \operatorname{sign}(\varphi), \quad y=\varphi,
$$

where $J$ is the moment of inertia of the torpedo and $\varphi$ is its angle of rotation. For this example, the problem of diagnosis of a single fault in the parameter space was simulated in Simulink using two algorithms, for independent observers and interacting observers matched with technical states. The intermittent fault in the form of the sequence of deviations of the parameter $C_{1}$ from the nominal value and returns to this value (first diagrams in Figures 6(a) and 6(b)) was simulated. The sinusoidal signal with an amplitude of 0.5 was fed to the input of the system. The second algorithm demonstrated the highest efficiency. Figure 6(b) shows the corresponding results of simulation (the confidence coefficients $K_{0}$ and $K_{1}$ for the serviceable and faulty technical states) of the diagnostic problem. It can be seen that, unlike the case of independent observers (Figure 6(a)), the diagnostic tools form the values of the confidence coefficients adequate to the real technical states. In order to quantitatively estimate the degree of adequacy of the results in both cases, the obtained realizations were used to calculate the probabilities of erroneous diagnosis. In this case, time intervals on which the system was faulty were analyzed. For these intervals, the total duration of subintervals on which erroneous signal on the serviceable state was formed (the confidence coefficient for the serviceable state reached the defined threshold value $A=0.9)$ was calculated. The resulting probability was determined as the ratio of this quantity and the total duration of the considered faulty intervals. Thus, for the method with independent observers, we obtained $P=0.91$, and for the method with interacting observers, $P=0.2$.

\section{Conclusions}

In this paper, we proposed the method of diagnosis of dynamic systems based on the application of the bank of fuzzy interacting state observers. We considered successively three different models of fault: structural changes, faults in the signal space, and faults in the parameter space. For these models of fault, we considered various structures of diagnostic tools different in the organization of the bank of the state observers and decision making rules. The proposed method was compared with the known method with independent observers by simulation in Simulink. As a result, it was demonstrated that the proposed method makes it possible to achieve higher quality of diagnosis.

\section{Acknowledgment}

This work was supported by the Russian Foundation for Basic Research, Project no. 10-08-00035a.

\section{References}

[1] R. J. Patton, P. M. Frank, and R. N. Clark, Issues in Fault Diagnosis For Dynamic Systems, Springer, London, UK, 2000.

[2] R. Isermann, "Model based fault detection and diagnosis-status and applications," in Proceedings of the 16th IFAC Symposium on Automatic Control in Aerospace, pp. 150-157, St. Petersburg, Russia, 2004.

[3] A. N. Zhirabok, "Robust observer design: logic-dynamic approach," in Proceedings of the 7th IFAC Symposium on Fault Detection, Supervision and Safety of Technical Processes, pp. 786791, Barcelona, Spain, July 2009.

[4] M. Benini, M. Bonfè, P. Castaldi, W. Geri, and S. Simani, "Design and analysis of robust fault diagnosis schemes for a simulated aircraft model," Journal of Control Science and Engineering, vol. 2008, Article ID 274313, 18 pages, 2008.

[5] J. L. Mata-Machuca, R. Martinez-Guerra, and H. Aguilar-Sierra, "Fault estimation using a polynomial observer: a real-time application," in Proceedings of the 8th IFAC Symposium on Fault Detection, Supervision and Safety of Technical Processes (SAFEPROCESS '12), pp. 552-557, Mexico City, Mexico, August 2012.

[6] O. Adrot and J. M. Flaus, "Fault detection based on uncertain switching models with bounded parameters," in Proceedings of the 7th IFAC International Symposium on Fault Detection, Supervision and Safety of Technical Systems (SAFEPROCESS'09), pp. 774-779, Barcelona, Spain, July 2009.

[7] H. Rios, C. Edwards, J. Davila, and L. Fridman, "Fault detection and isolation for nonlinear systems via HOSM multipleobserver," in Proceedings of the 8th IFAC Symposium on Fault Detection, Supervision and Safety of Technical Processes (SAFEPROCESS '12), pp. 534-539, Mexico City, Mexico, August 2012.

[8] M. Muenchhof, "Multi-model based fault detection and diagnosis of a hydraulic servo axis," in Proceedings of the 17th World Congress The International Federation of Automatic Control, p. 13117, Seoul, Korea, July 2008.

[9] J. Davies, H. Tsunashima, R. M. Goodall, R. Dixon, and T. Steffen, "Fault detection in High Redundancy Actuation using an interacting multiple-model approach," in Proceedings of the 7th IFAC International Symposium on Fault Detection, Supervision and Safety of Technical Systems (SAFEPROCESS'09), pp. 12281233, Barcelona, Spain, July 2009.

[10] S. P. Dmitriev, N. V. Kolesov, and A. V. Osipov, Information Reliability, Control, and Diagnosis of Navigation Systems, TSNII Elektropribor, Saint-Petersburg, Russia, 2003.

[11] J. M. Koscielny, "Application of fuzzy logic for fault isolation in a three tank system," in Proceedings of the 14th World Congress International Federation of Automatic Control (IFAC '99), vol. 7, pp. 73-78, Beijing, China, 1999.

[12] L. F. Mendouca, J. M. Sousa, and J. M. G. Sida Cos, "Fault isolation using fuzzy model based observers," in Proceedings of the 6th IFAC Symposium on Fault Detection, pp. 142-150, Beijing, China, 2006. 
[13] A. Akhenak, M. Chadli, J. Ragot, and D. Maquin, "Design of observers for Takagi-Sugeno fuzzy models for fault detection and isolation," in Proceedings of the 7th IFAC International Symposium on Fault Detection, Supervision and Safety of Technical Systems (SAFEPROCESS'09), pp. 1109-1114, Barcelona, Spain, July 2009.

[14] Yu. N. Andreev, Control of Finite Dimensional Linear Objects, Nauka, Moscow, 1976. 

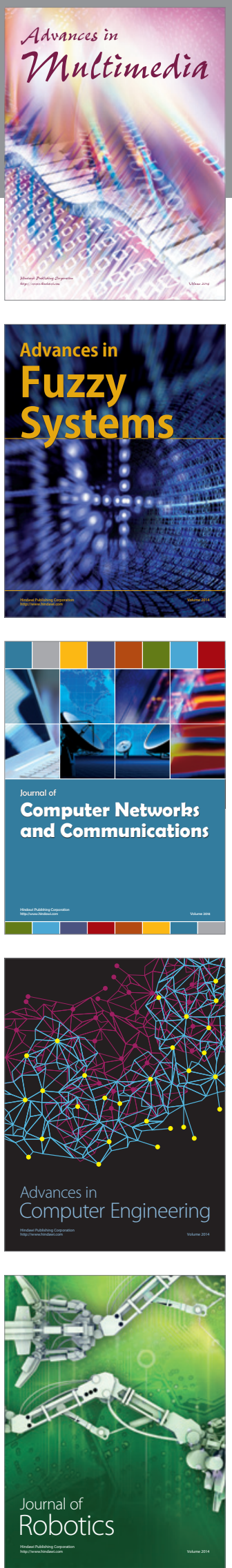

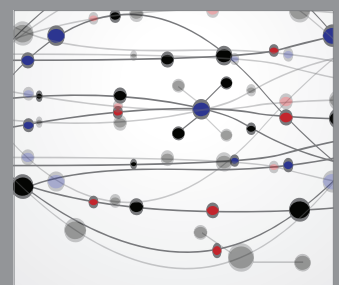

The Scientific World Journal
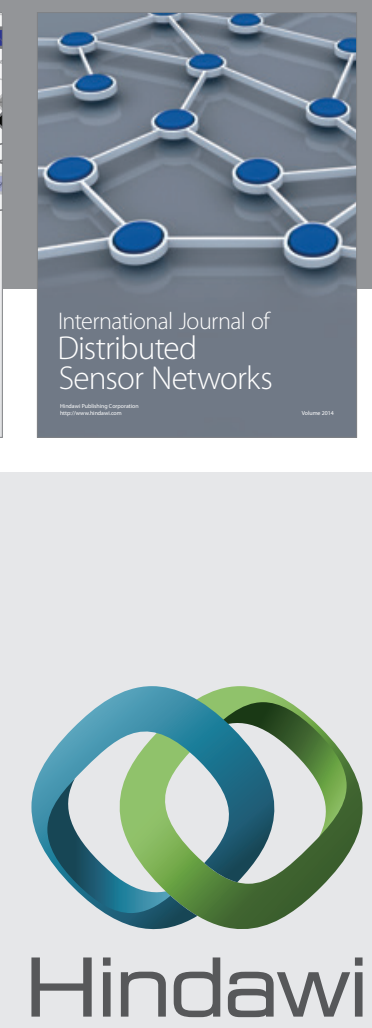

Submit your manuscripts at

http://www.hindawi.com
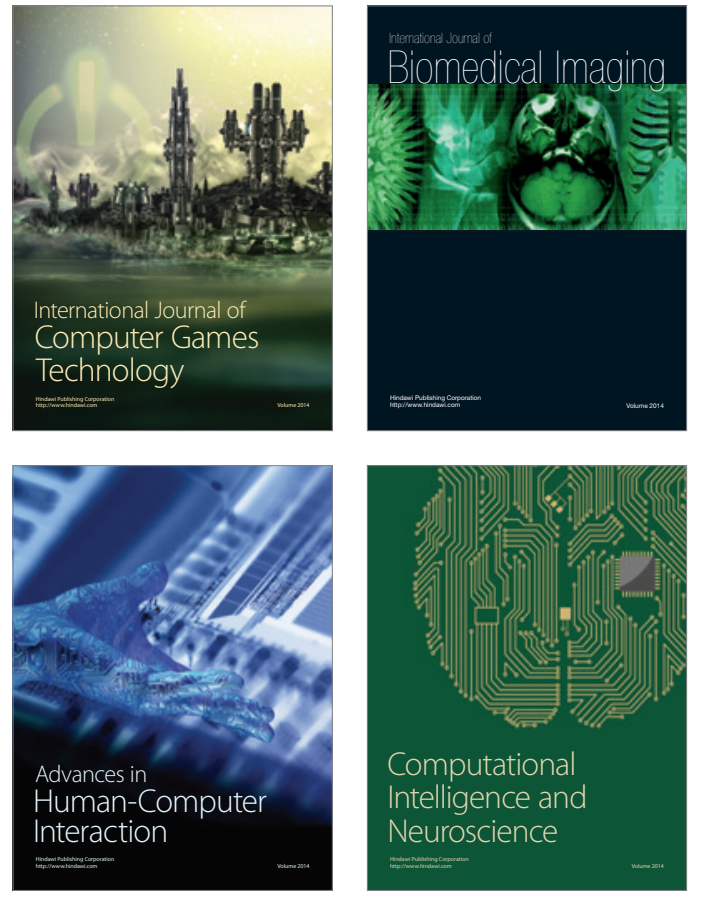
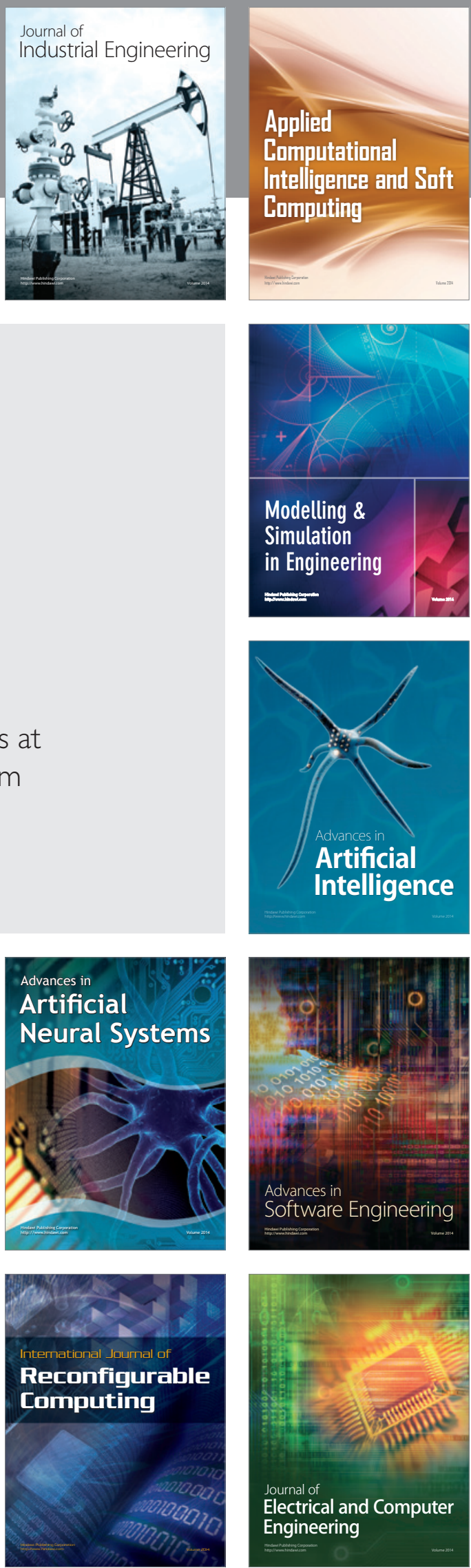